\title{
An experimentally verified method to predict \& measure all forms of Cyber-Risk
}

\author{
Riccardo C. Storti ${ }^{i}$ \\ Chief Engineer, Delta Group Engineering, Melbourne, Australia
}

Saturday, March 20, 2021

\begin{abstract}
A precise \& unambiguous mathematical definition of Cyber-Risk is developed, yielding an experimentally validated solution demonstrating 'How to Predict \& Measure Cyber-Risk' for any Internet Connected Information System (ICIS) to greater than 98.07\% accuracy. Moreover, it is shown that the solution holds for all scales of ICIS, from an Application level to an Enterprise level. In addition, it is shown that Test Effort Estimation (TEE) quantifies Cyber-Confidence, which in turn quantifies Cyber-Risk. Hence, TEE is a Mission Critical Activity (MCA) when formulating Cyber-Risk Management Strategies \& may be utilised prior to project commencement, in-flight or post facto as an assessment \&/or auditing tool. The TEE Model Construct developed is a statistical based methodology whereby the evaluations/decisions made, result in the contraction or expansion of the ' $z$-Score' associated with an infinite population of database records. The primary advantage of this approach is that very little information is required client-side at the engagement stage in order to produce peer acceptable estimates of the required test effort, \& to accurately predict \& measure the associated Cyber-Risk. This approach empowers clients \& service providers to precisely define whatever level of Cyber-Risk is to be contractually delivered, capable of being absorbed, or prepared to be absorbed by consensus. With the aid of a decision table, estimators are able to articulate \& convey to the appropriate authorities, various levels of Cyber-Risk commensurate with the available resources. The TEE Model Construct developed, presents an experimentally verified methodology, cognizant of commercial realities, yielding the following key advantages; (i) it requires minimal inputs, (ii) it has a scientific foundation, (iii) it facilitates operational decision-making, (iv) it quantifies Risk Based Testing (RBT), (v) it is simple, robust, flexible, consistent, reusable \& transparent, (vi) it is capable of scaling a projected solution from a known solution, (vii) it embraces Continuous Improvement Processes (CIP's), (viii) it confines perceptual subjectivity predominantly to three variables \& (ix), it commercially exists as an off-theshelf product.
\end{abstract}

Key Words: Applied Probability, Cyber-Confidence, Cyber-Risk, Decision-Facilitation, Estimation, Operations, Quality Assurance, Risk Analysis, Software Testing

i Delta Group Engineering; rstorti@gmail.com; ORCID-ID: 0000-0002-0152-3888 


\section{Introduction}

\subsection{Solution Background}

In this article, we investigate the corporate Cyber-Risk associated with 'being connected' to a large information network. The Cyber-Risk Prediction \& Measurement Construct presented herein has been experimentally validated to greater than $98.07 \%$ accuracy, \& all experimental results are available for peer review [1]. The solution presented within this article is commercially available \& has undergone a rigorous development \& validation regime prior to commercial use.

\subsection{Summary of Results}

The objective of the solution presented is to predict the amount of testing required for any application or system; e.g. building an application to 99(\%) Defect-Free Confidence. For such an objective, the only unbiased means by which to validate the outcome is to test the predictive model against an Application of its own creation; that is, to build an Application which predicts \& measures Cyber-Risk, then validating what was built against the Mathematical Model predicting the outcome, months in advance. Attempting to utilize commercial clients to validate predictions was not feasible due to the commercial nature of engagements. Nameless client results could have been presented but they cannot be confirmed by peer review due to commercial sensitivity. Therefore, the only verifiably unbiased means by which to validate our solution is available for peer review [1].

The solution was initially developed as a Mathematical Model utilizing a sophisticated Engineering Application [2], [3]. Once the Engineering Model was completed, it was assembled as an Experimental Prototype in spreadsheet form. A Developer was engaged $\&$ the Experimental Spreadsheet Prototype issued. The purpose of the engagement was to construct a Web-Application capable of performing identical calculations to those appearing in the Experimental Spreadsheet Prototype. No additional Requirements documentation was provided to the Developer, \& the Application as it appears in its current form, was designed \& built ad-hoc via Skype sessions; the project was executed utilizing Agile Methodology. No bias or consideration was undertaken to artificially guide the completed solution such that the number of tests required to validate it coincided with predictions. In other words, the experimental evidence gathered \& confirmed is genuine \& cannot be considered questionable by any measure. A summary of our results appears in Tab. (1) as follows;

\begin{tabular}{|c|c|c|c|}
\hline DIT Limit & Predicted & Measured & Similarity \\
\hline Upper & 3,318 & 3,254 & $>98.07 \%$ \\
\hline Lower & 126 & 143 & $>88.11 \%$ \\
\hline
\end{tabular}

Tab. (1): Predicted DIT's \& Measured Results

Where:

- $\quad$ DIT = Dynamic Information Test 


\subsection{Simplified Examples}

An effective means by which to communicate the decision making power of the solution presented herein is to answer some obvious questions utilising several simplified examples, as follows;

1. Cyber-Security Confidence against Port Attack

2. Cyber-Security Confidence against Brute Force Attack

3. Cyber-Risk associated with Internet Banking

4. 'Splunk $>$ Phantom'

\subsubsection{Port Attack}

If all Transmission Control Protocol (TCP) \& User Datagram Protocol (UDP) Ports are tested \& confirmed to be inactive, how secure is my computer against penetration from external actors according to Open Web-Application Security Project (OWASP) Test Scenarios?

- Answer: Cyber-Security Confidence $\mathbf{= 9 9 . 8 3 \%}$ as shown in Fig. (1) Where:

- The number of DIT's = the number of Ports tested

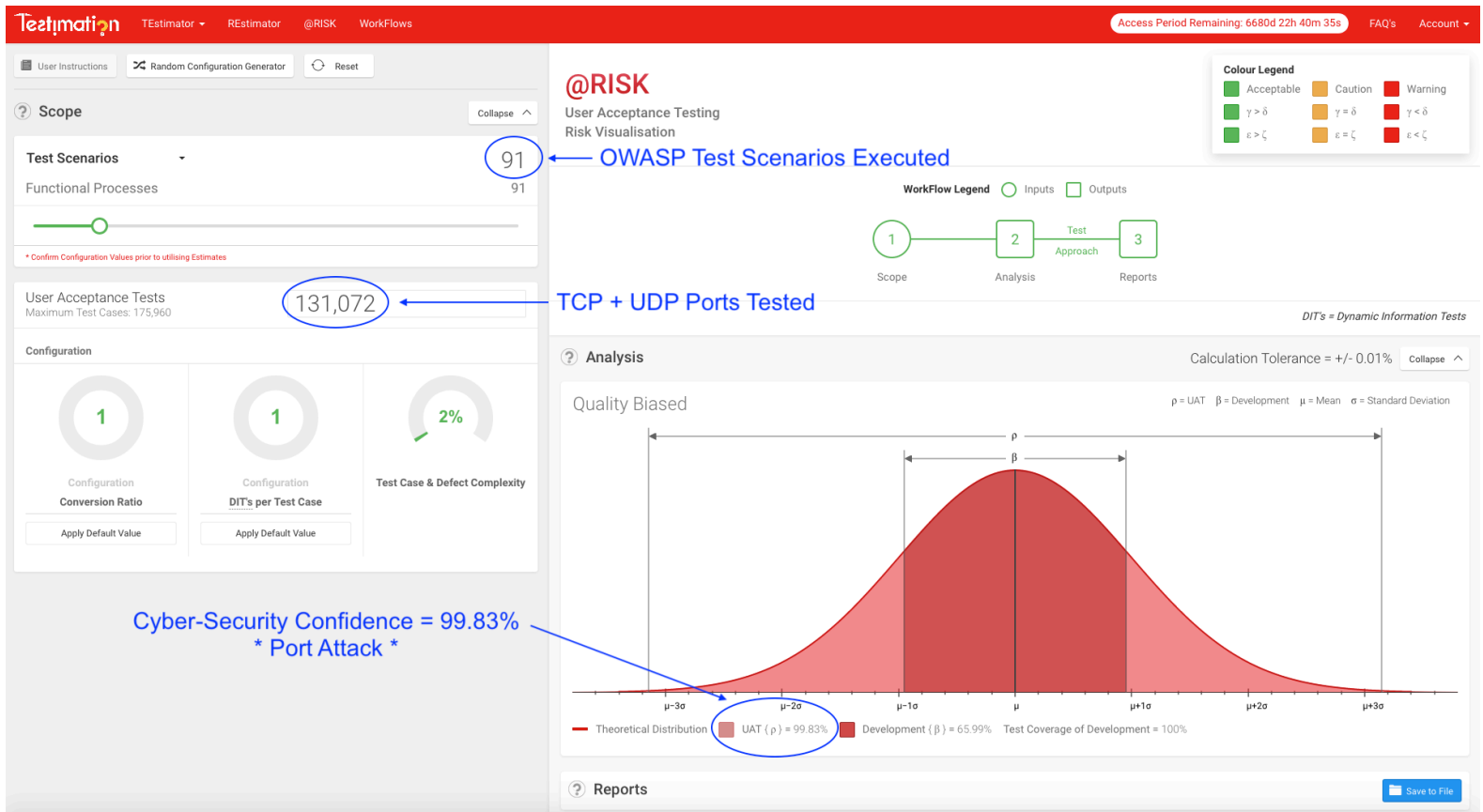

Fig. (1): Cyber-Security Confidence Measurement (Port Attack)

\subsubsection{Brute Force Attack}

What is the Cyber-Security Confidence associated with a Brute Force Attack on a User Login Function, utilising the Oxford English Dictionary as the Test Basis?

- Answer: Cyber-Security Confidence $\mathbf{9 9 9 . 9 7 \%}$ as shown in Fig. (2) Where:

- The number of DIT's = the number of words in the Oxford English Dictionary 


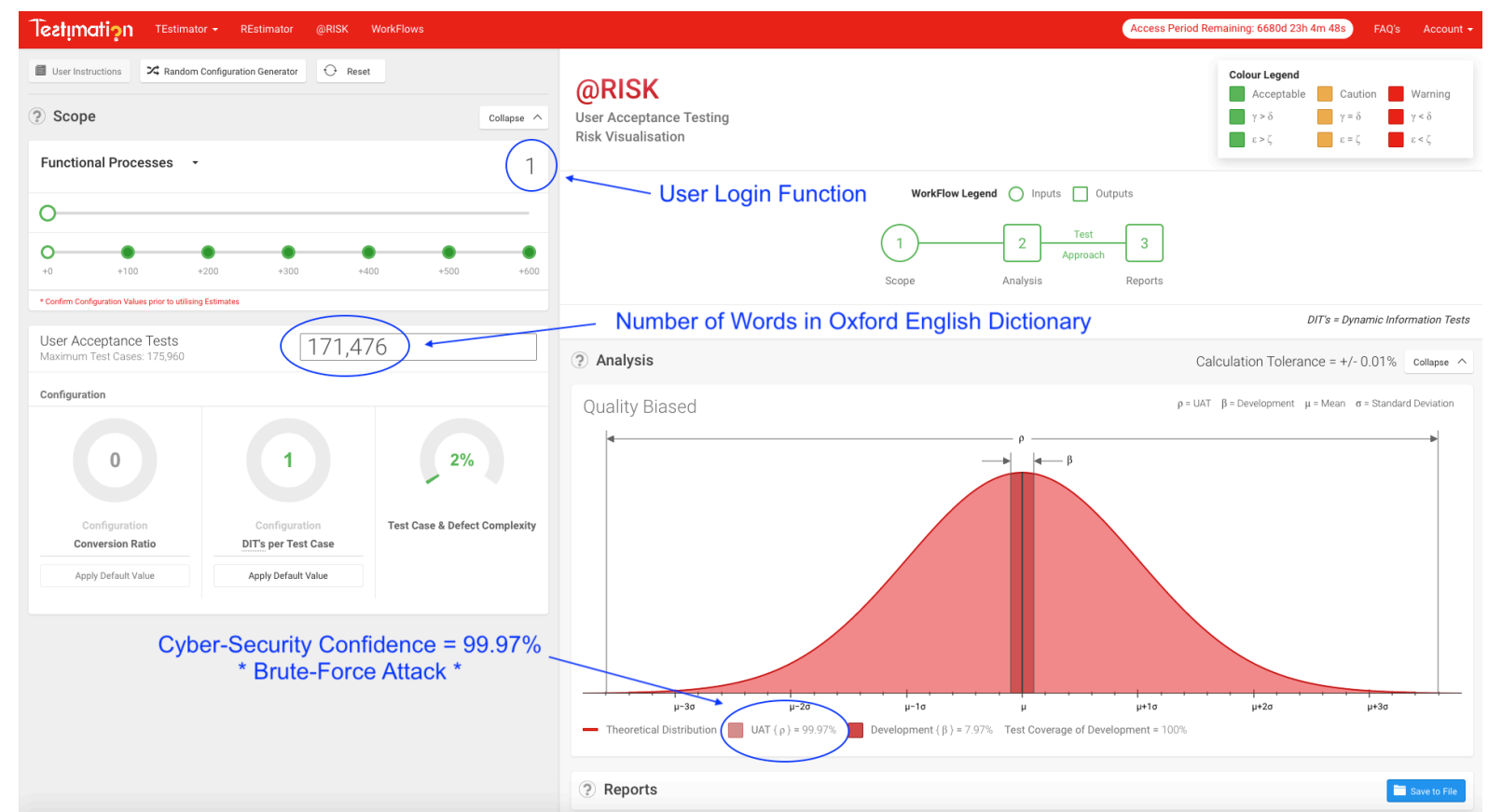

Fig. (2): Cyber-Security Confidence Measurement (Brute Force Attack)

\subsubsection{Internet Banking}

How many Test Cases are required in order to deliver Internet Banking Functionality satisfying the conditions 'Cyber-Risk $\leq 1 \%$ ' \& 'Cyber-Confidence $\geq 99 \%$ '?

- Answer: Number of Test Cases $=\mathbf{3 , 1 8 5}$ as shown in Fig. (3)

Where:

- The number of User Pathways (UP's) through the Graphical User Interface $=120$

- The number of DIT's per Test Case = the number of Test Steps per Test Case

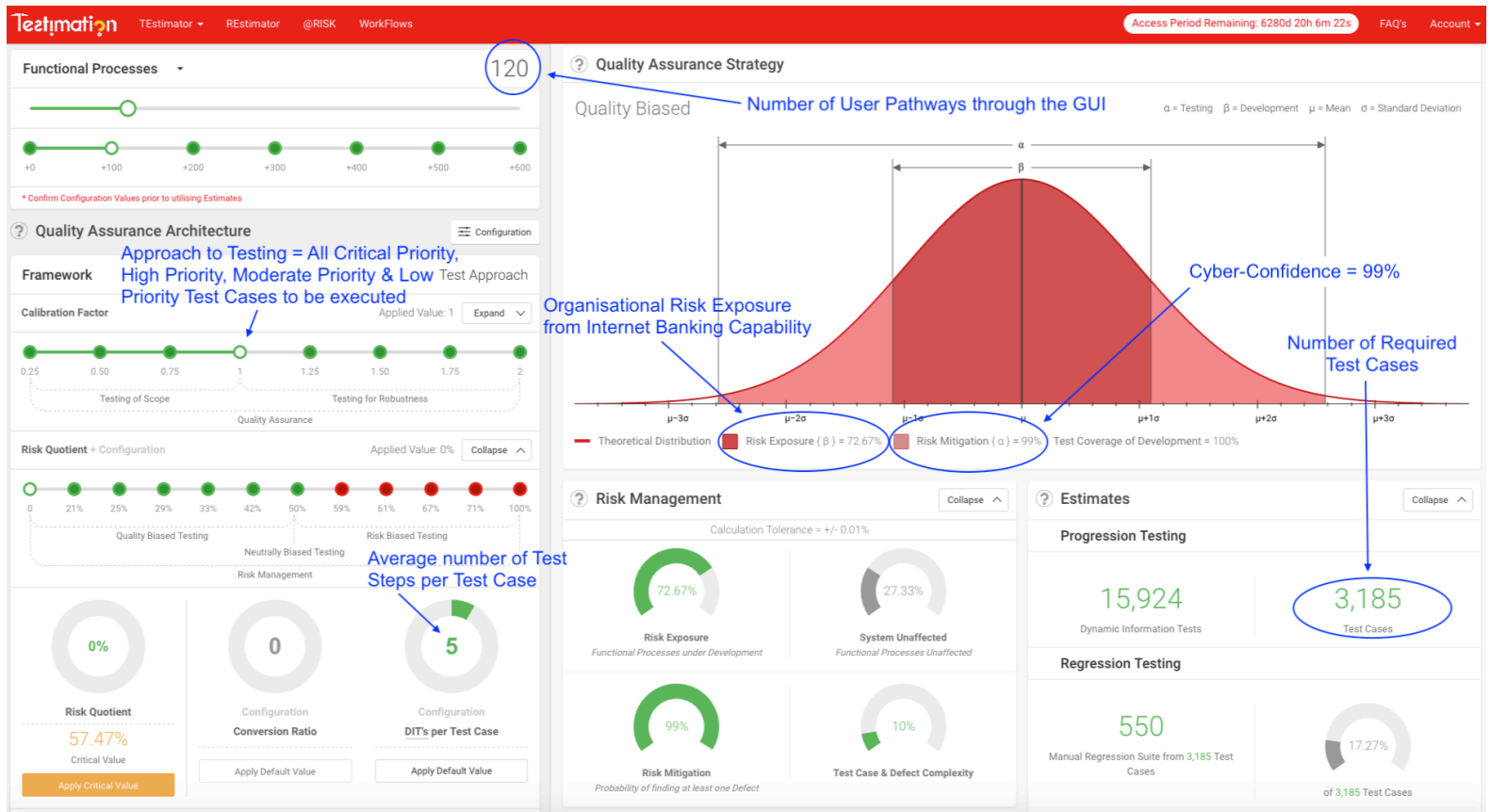

Fig. (3): Cyber-Confidence Measurement (Internet Banking) 
Thus, designing \& executing 3,185 Test Cases with an average of five (5) Test Steps per Test Case yields a 99\% probability that the deployed solution is Defect-Free if all Test Cases pass.

\subsubsection{Splunk>Phantom}

'Splunk>Phantom' is a Cyber-Security Workflow Automation Tool (WAT). In this example, a Security Operations Centre (SOC) is transitioning to the 'Splunk>Phantom' platform, but the SOC-Team has limited Quality Assurance (QA) experience. How many Test Cases do they need to execute in order to test the Playbook shown in Fig. (4), prior to deploying the automated Cyber-Security solution?

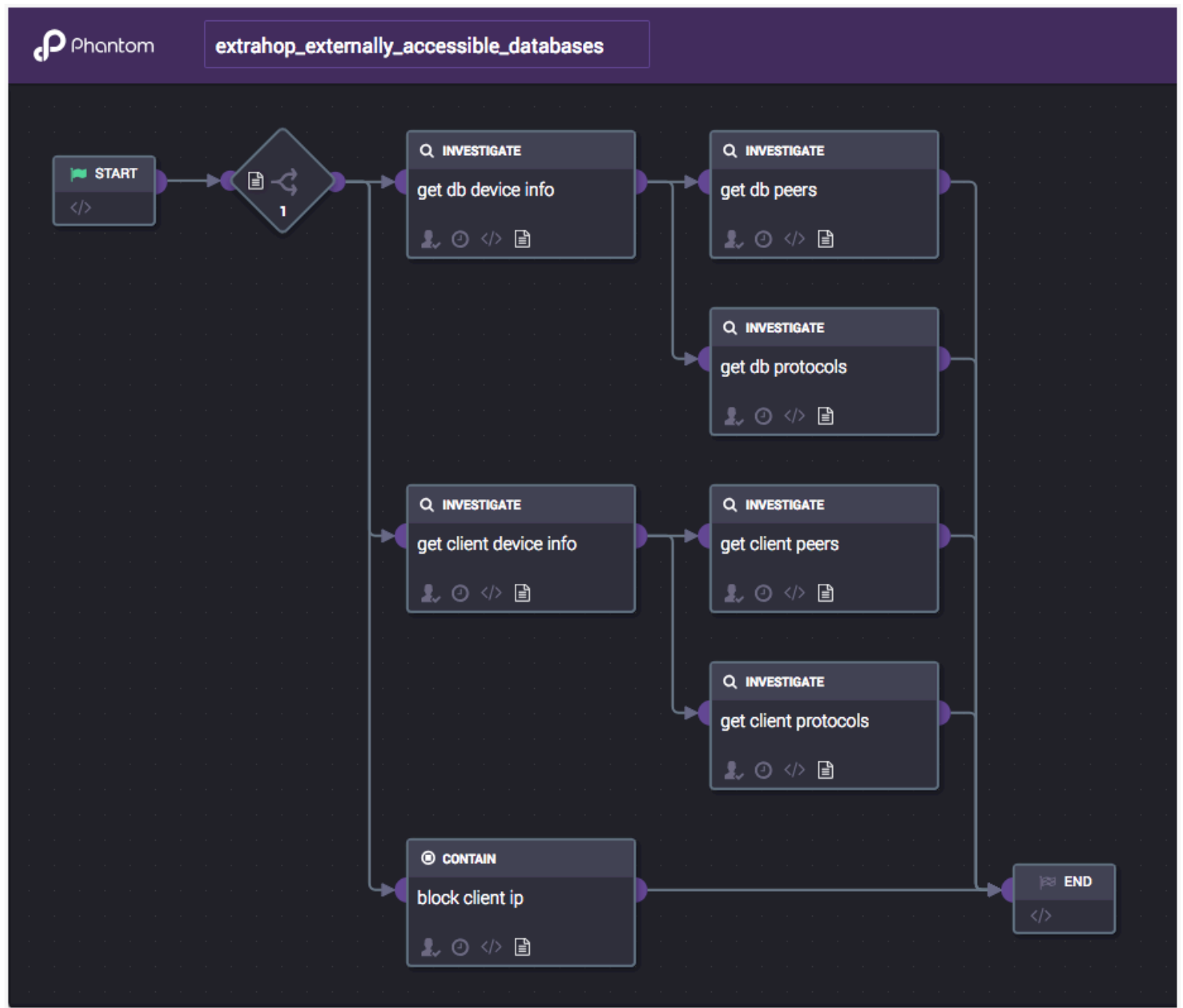

Fig. (4): Example 'Splunk>Phantom' Playbook

Fig. (4) Playbook Facts:

1. Five (5) Informational Flow Pathways (IFP's) are drawn from Start-to-End

2. The SOC-Team have estimated or counted an average of seventeen (17) Acceptance Criteria per IFP

3. One (1) Acceptance Criteria = One (1) DIT 
Utilising The Cyber-Risk Prediction \& Measurement Construct presented herein, we may formulate a Decision Assistance Table (DAT) as shown in Tab. (2);

\begin{tabular}{|c|c|c|c|c|}
\hline QA-Solution & Cyber-Confidence & Cyber-Risk & Test Cases & Test Cases per IFP \\
\hline 1 & $0 \%$ & $100 \%$ & 0 & 0 \\
\hline 2 & $63.27 \%$ & $36.73 \%$ & 5 & 1 \\
\hline 3 & $80.22 \%$ & $19.78 \%$ & 10 & 2 \\
\hline 4 & $93.15 \%$ & $6.85 \%$ & 20 & 4 \\
\hline 5 & $97.43 \%$ & $2.57 \%$ & 30 & 6 \\
\hline 6 & $99 \%$ & $1 \%$ & 40 & 8 \\
\hline 7 & $99.6 \%$ & $0.4 \%$ & 50 & 10 \\
\hline 8 & $99.84 \%$ & $0.16 \%$ & 60 & 12 \\
\hline 9 & $99.93 \%$ & $0.07 \%$ & 70 & 14 \\
\hline 10 & $99.97 \%$ & $0.03 \%$ & 80 & 16 \\
\hline
\end{tabular}

Tab. (2): Fig. (4) DAT

Tab. (2) demonstrates that Test Effort increases, as Cyber-Risk tends to zero; so, which QA-Solution should the SOC-Team apply? To answer this question, the SOC-Team need to recognise that each IFP from any Playbook requires a minimum of two Test Cases; one testing for success \& one testing for failure; i.e. one Positive \& one Negative Test Case respectively, thus QA-Solution $(1,2)$ may be eliminated from consideration.

In many commercial environments, QA-Teams are often pressured into minimal testing solutions. To overcome this challenge, we may utilise Fig. (5) to specify the optimal QASolution from Tab. (2). In Fig. (5), we see that dimension ' $\alpha$ ' is much greater than dimension ' $\beta$ ' $(\alpha>>\beta)$ for QA-Solution (3). Hence, the Risk Mitigation is much greater than the organisational Risk Exposure associated with the transition from manual to automated Workflows. Therefore, QA-Solution (3) denotes the optimal target such that:

- Risk Exposure $=17.69 \%$ O Organisational Impact of Workflow Changes

- Risk Mitigation $=80.22 \%=$ Cyber-Confidence $\equiv$ Test Coverage

- Cyber-Risk $=19.78 \% \equiv$ Testing Not Executed

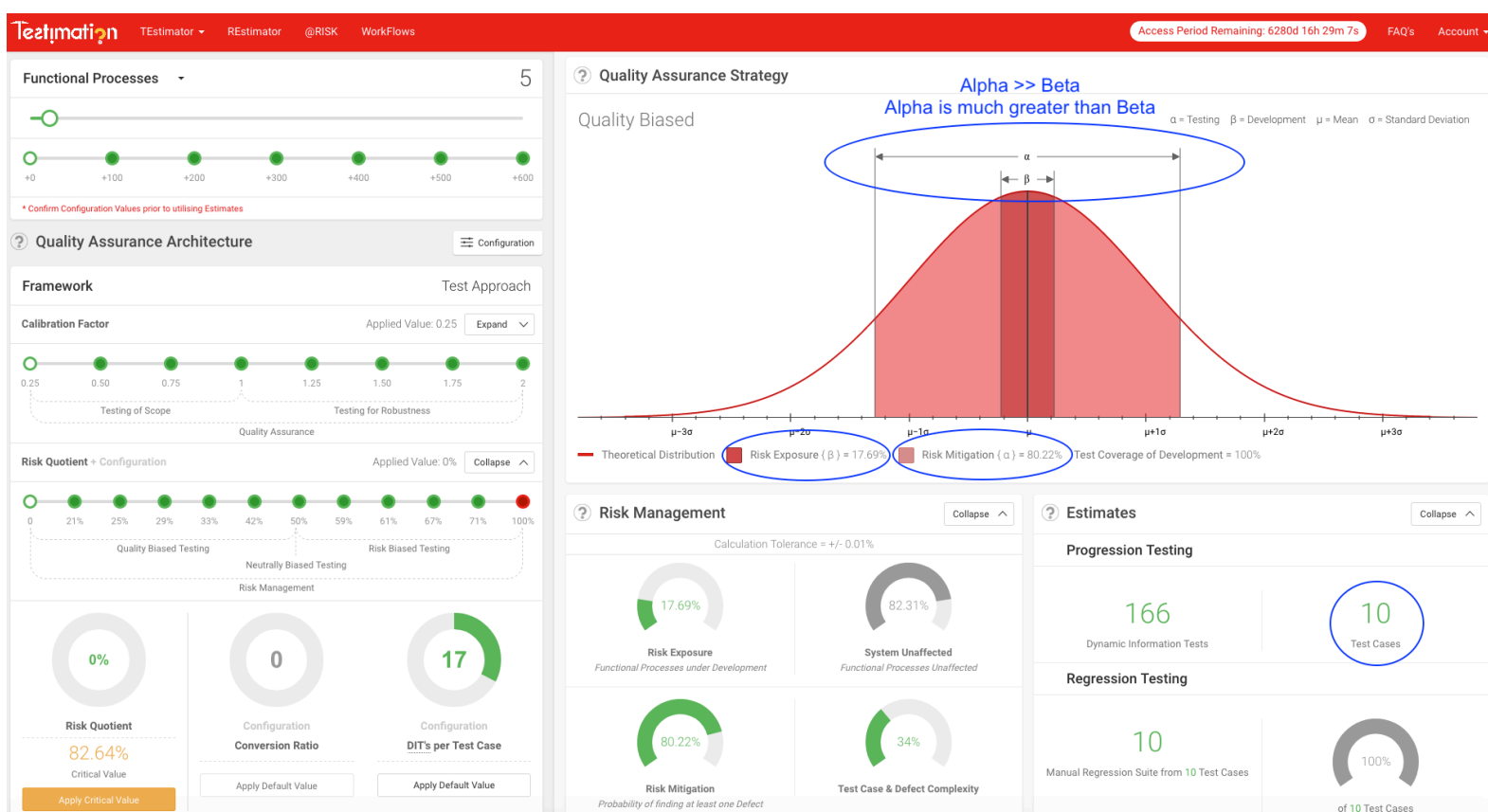

Fig. (5): Cyber-Confidence Measurement ('Splunk>Phantom' Playbook) 


\section{Defining The Problem}

\subsection{Dilemma}

The evolution of The Internet has brought great convenience to many of us. The perceived need for staying 'connected' is beyond measure \& seems insatiable. The meteoric rise of The Internet has prompted many governments around the world to abandon traditional means of initiating dispute resolution, in favour of information gathering media such as social platforms. Even password rules have adopted the requirement for special characters (e.g. \#), influenced by the introduction of social media hash tags. Whether or not the rise-\&-rise of The Internet bares a Net Positive Value in the long term is a tale-best-told by the historians of the future. However, with convenience \& connectivity comes the peril of abuse. As time marches forward, the potential for mishap (accidental or malicious) is synchronous with our exponential demand for connectivity \& functionality, hence our dilemma; how do we manage the associated Risk (termed Cyber-Risk)?

\subsection{The Information Universe}

Prior to resolving our dilemma, we must first conceptualise the environment in which we seek to manage the associated Cyber-Risk. To achieve this, we shall utilise Cosmology as an analogous working guide. The Physical Universe \& The Information Universe (i.e. The Internet) share some important common characteristics as follows:

1. The Physical Universe possesses an undefined boundary; thus, its information superset cannot be precisely quantified \& is considered to be infinite:

- Similarly, the boundary of The Internet is undefined \& may be considered to contain an infinitely large information superset

2. All matter in The Physical Universe is gravitationally connected. Matter is information, which interacts with other sources of information at multiple scales of reality, either directly or indirectly:

- Similarly, all information on The Internet is connected, directly or indirectly, via the Functional Processes contained within all forms of software

3. The Big-Bang was an explosion of Space-Time; thus, the centre of The Physical Universe is everywhere:

- Similarly, the centre of The Internet is everywhere; i.e. it coincides with all forms of User Access (e.g. any smart device, any computer etc.) simultaneously

The points above are critical to embrace; we shall extensively exploit these parallels to predict \& measure Cyber-Risk by recognising a key mathematical proof:

- All infinite populations are (by some measure) Normally Distributed; hence, for an infinitely large population of database records, the number of populated fields within the information superset will obey a Normal Distribution

\subsection{What is Cyber-Risk?}

Numerous concepts regarding the nature of Cyber-Risk exist. The most commonly held perception being that Cyber-Risk is the Risk Exposure associated with external actors attempting to penetrate IT-Systems. This of course is partially correct, but the CyberRisk associated with system penetration is not the only form of Cyber-Risk. Alternative forms of Cyber-Risk also exist \& are equally concerning; e.g. coding errors, system 
performance, system response to stress, incorrect Business Processes etc. However, herein we define Cyber-Risk as the precise \& unambiguous mathematical relationship given by Eq. (1) as follows:

$$
\text { Cyber-Risk = } 1 \text { - Cyber-Confidence }
$$

Where, for Internet Connected Information Systems (ICIS's):

1. Cyber-Confidence $=$ The number of tests which have actually been executed \& passed, expressed as a statistical probability

2. Failed tests = Identified Defects; these are Cyber-Issues, not Cyber-Risks

3. Thus, by mathematical definition, for all forms of Cyber-Risk:

- Cyber-Risk $=$ The number of possible tests which have not been executed, expressed as a statistical probability

Recognising that:

4. Testing Not Executed $\equiv$ Undiscovered Defects

Consequently yields the following conclusions:

5. The natural ally of Cyber-Confidence is Quality Assurance (QA)

6. The required level of Cyber-Confidence to be delivered by a QA-Team is determined in the Test Effort Estimation (TEE) phase

7. The expected level of Cyber-Risk may be quantified at an early stage in the project lifecycle, in-flight or post facto; hence, Eq. (1) denotes a powerful decision making tool

- TEE quantifies Cyber-Confidence, which in turn quantifies Cyber-Risk, as shown by Eq. (1). Hence, TEE is a Mission Critical Activity (MCA) when formulating Cyber-Risk Management Strategies \& may be utilised prior to project commencement, in-flight or post facto as an assessment \&/or auditing tool

\subsection{Experimental Validation}

The Cyber-Risk Prediction \& Measurement Construct presented herein has been experimentally validated to greater than $98.07 \%$ accuracy, where:

1. All experimental results are available for peer review [1]

2. The stepwise Mathematical Modeling of The Engineered Solution is available for peer review [2]

3. A Case Study is available for peer review, demonstrating the following [3]:

- How we delivered a project $30 \%$ cheaper \& $15 \%$ faster without increasing manning levels

- How Over-Testing \& Under-Testing occurs

Therefore:

- How Quality-Gaps are formed

4. Eq. (1) is 'Fit-for-Purpose' to formulate Cyber-Risk Management processes

\subsection{What is Cyber-Risk Management?}

In the preceding sections, we precisely defined Cyber-Risk utilizing Eq. (1). We have also referenced experimentally validated evidence of all proposed claims, available for peer review [1], [2]. Consequently, our solution offers the following Cyber-Risk Management capabilities:

1. Continuous (real time) re-estimation \& re-evaluation 
2. Standardisation, transparency, repeatability \& objectivity

3. Predictive probability of finding defects prior to project commencement, hence:

- Minimising Cyber-Risk \& maximising Cyber-Risk Governance

- Providing clear delivery targets to service providers

- Providing a contractual basis (specification) for service delivery

4. Predictive probability of residual defects within systems (real time), hence:

- Can be applied to proportion accountability for defects resulting in \$ loss

5. A scientific platform 'for or against' litigation

These capabilities may be summarized \& categorized into three key areas of Predictive Business Driven Governance (PBDG), in accordance with Tab. (3) as follows;

\begin{tabular}{|c|c|c|}
\hline \multirow{10}{*}{ PBDG } & Capability & Description of predictive capability \\
\hline & \multirow{2}{*}{$\begin{array}{c}\text { Quality } \\
\text { Assurance }\end{array}$} & Minimum number of required tests \\
\hline & & Maximum number of required tests \\
\hline & \multirow{5}{*}{$\begin{array}{l}\text { Cyber-Risk } \\
\text { Management }\end{array}$} & $\%$ Probability of finding defects \\
\hline & & \% Risk Exposure \\
\hline & & $\%$ Code changes covered by testing \\
\hline & & \% System covered by User Acceptance Testing (UAT) \\
\hline & & \% Level of Quality Assurance (QA) provided by $3^{\text {rd }}$ parties \\
\hline & \multirow{2}{*}{$\begin{array}{c}\text { Project } \\
\text { Management }\end{array}$} & Testing man-day effort \\
\hline & & Project budgeting \\
\hline
\end{tabular}

Tab. (3): Predictive Business Driven Governance Capability

We are now positioned to define Cyber-Risk Management in terms of Business Driven Processes (BDP's) as expressed in Fig. (6, 7), incorporating a transposed form of Eq. (1);

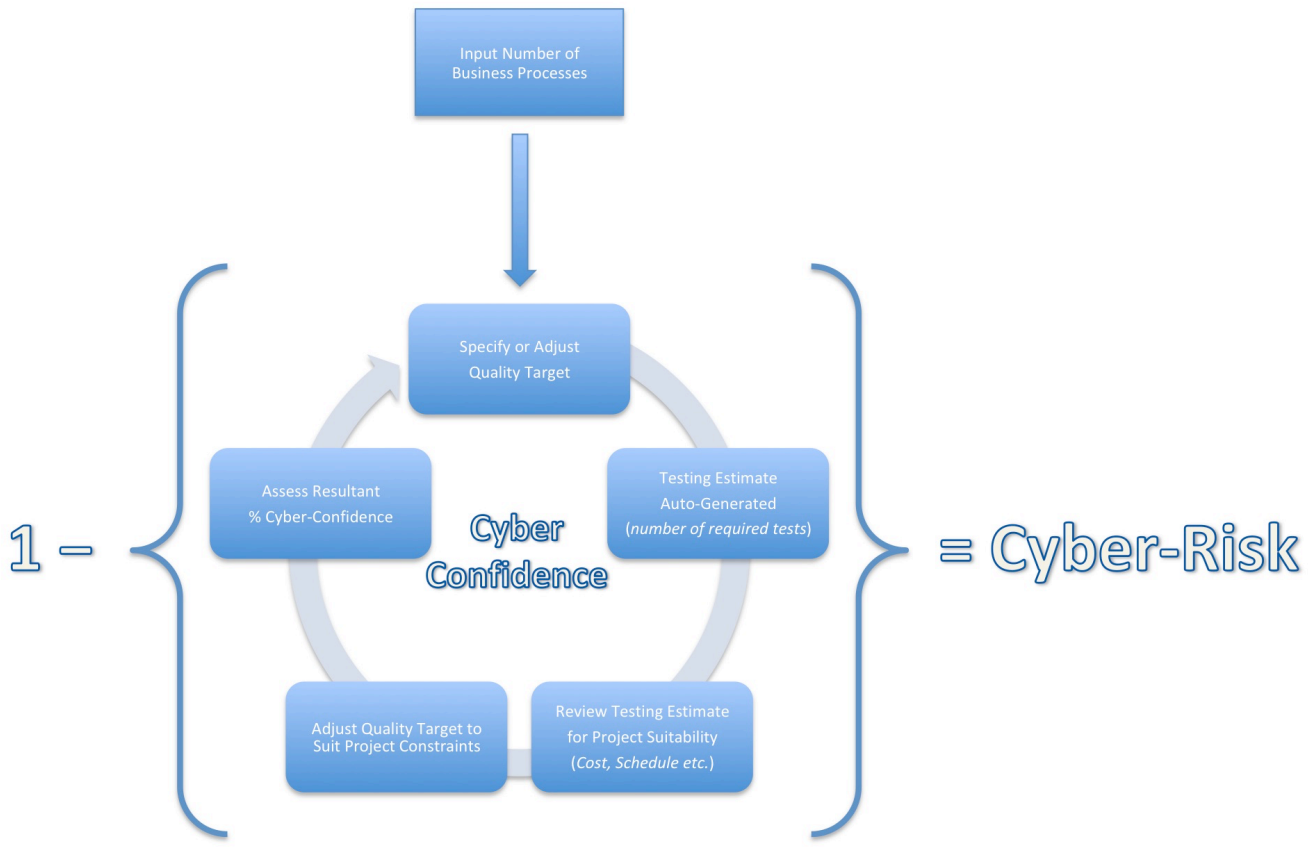

Fig. (6): Business Driven Cyber-Risk Management Process (Continuous)

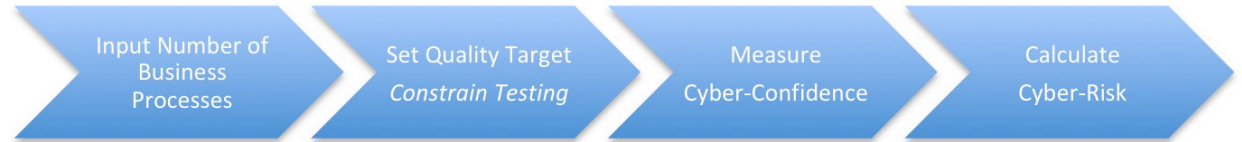

Fig. (7): Business Driven Cyber-Risk Management Process (Lineal) 
Ideally, the BDP's specified in Fig. $(6,7)$ should be supported by the hierarchical structure shown in Fig. (8);

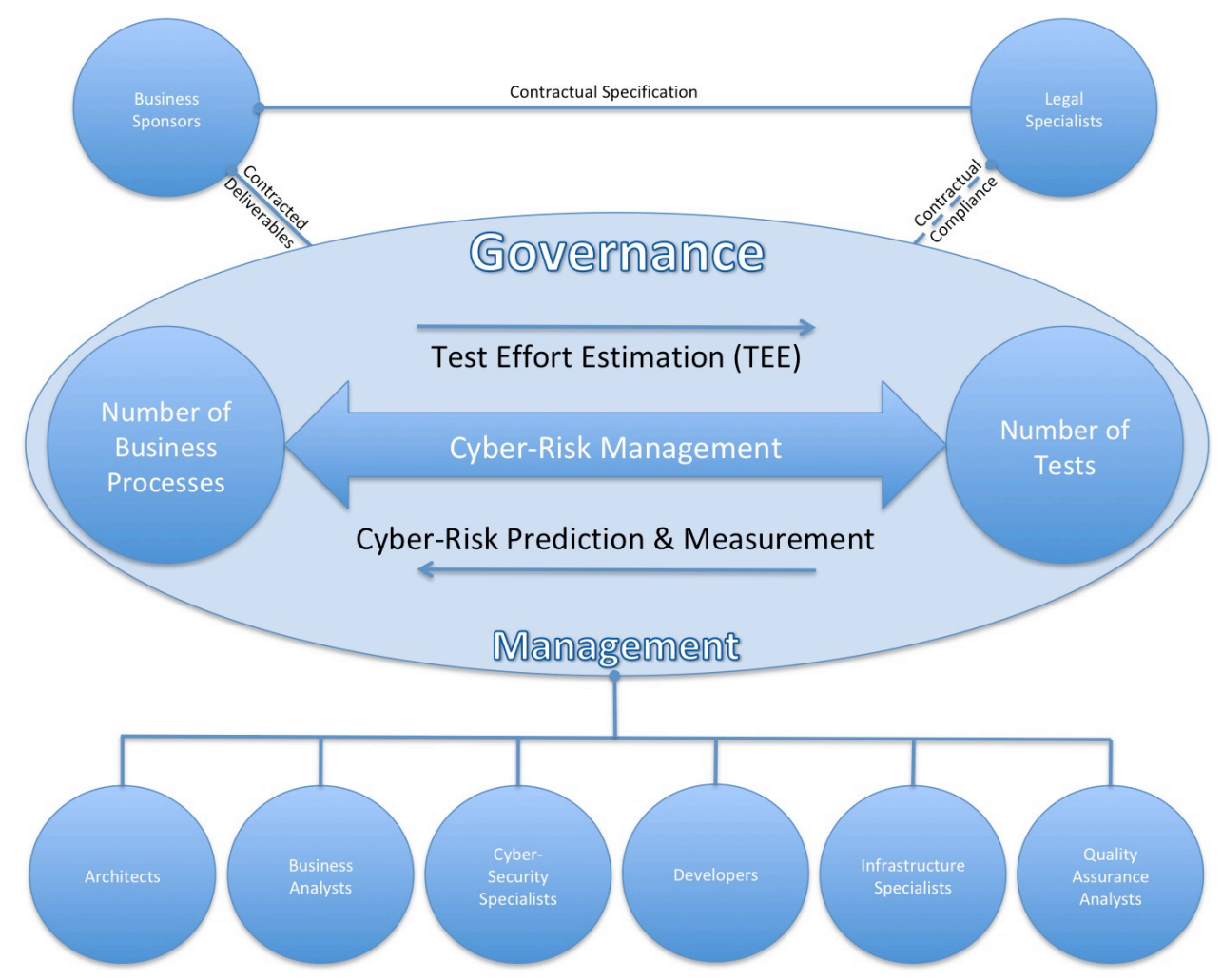

Fig. (8): Business Driven Cyber-Risk Management Hierarchy

\subsection{What is Quality Assurance?}

Numerous interpretations of Quality Assurance (QA) exist in the literature \& software development communities; on the surface, it seems to mean different things to different people. However, within the forum of this article, we shall define QA utilizing a contrasting paradigm as follows:

1. Testing $=$ the validation associated with a single unit (i.e. one test)

2. $\mathrm{QA}=$ the validation associated with a population of units (i.e. many tests)

\subsection{Client-Side Issues}

A broad spectrum of techniques exists in the public domain to assess \& estimate the effort required to test software [4], [5], [6], [7], [8], [9], [10], [11], [12], [13]. However, none of the literature cited includes any viable technique suitable to the early stages of commercial engagement. Upon completing a thorough literature review, one may be left with the impression that software occurs naturally, which sprung into existence at the instant of the Big-Bang (the birth of the Cosmos). One may be forgiven for concluding, based upon existing community literature, that software development never historically occurred from a zero-point, but has always existed \& was built upon a naturally occurring organic base. All the literature surveyed herein, \& indeed the literature reviewed by the authors beyond the citations defined, requires a level of knowledge for decision making substantially exceeding that available at the time of initial client engagement. Moreover, in much of the literature reviewed, individuals with 
development skill-sets, not specifically Quality Assurance (QA) skill-sets, formulate estimates. In addition, developers tend to assess \& estimate based upon technical or architectural perspectives, not from the only perspective of any commercial consequence; i.e. the perspective of the client, in terms they can comprehend. Commercial clients at the early stages of engagement are not primarily concerned with the number of logical statements distributed throughout code, nor are they concerned with Function Point's or screen layouts. Their primary concern orbits the guarantee from the service provider that their Business Processes (BP's) will work reliably in order for their core revenue streams to remain commercially viable. Unfortunately, for whatever relevant reasons, this fact has been largely ignored by software houses, in favour of more internally familiar concerns associated with difficulty \& complexity of coding.

Not only does the prevailing estimation wisdom fail for early stage engagements, it also biases software QA to defect finding exercises rather than the true objective of a commercial engagement; i.e. providing an agreed level of QA to the business \& sponsor, with respect to the solution to be delivered. A physical analogy to this is the Aluminium extrusion process. Aluminium sections when extruded, stretched \& cut to length are very often 'out-of-specification' with respect to straightness, twist, or a number of other physical parameters. These breaches in tolerance can occur for a variety of reasons (e.g. thermal variations during cooling), but the industry survives by client willingness to accept the product on a 'Fit-for-Purpose' basis. As long as an extrusion is not 'Out-ofSpecification' so much as to be unusable, it will often be accepted by the client rather than rejected; developing software is no different. As long as the core End-to-End (E2E) BP's are verified to function reliably so as not to impact revenue or the customer experience, clients are more likely to accept the solution delivered as opposed to 'fewer defects' accompanied by an increase in testing expenditure. Hence, it becomes very clear that for the majority of commercial engagements, finding defects should not be the objective of validating software. Rather, the emphasis should be on managing the associated Cyber-Risk.

\subsection{Vendor-Side Issues}

A critical element of the Software-Development-Life-Cycle (SDLC) involves estimating the magnitude \& scope of the effort required to validate the quality of the solution to be delivered to a client. This is such an important function that, if executed poorly, may lead to commercial losses for the project team as well as the client. For a service provider, accurate, reliable, consistent \& timely Test Effort Estimation (TEE) may determine commercial profitability. To emphasize this aspect of the SDLC, we may draw upon the analogy of a speculative builder in the Australian housing market. The commercial profitability of the speculative venture is often determined by the cost of the land, not the cost of construction. Construction costs are largely fixed by the economic climate, but a significant swing in expenditure potentially affecting profitability is the purchase price of the land required for the construction project. By analogy, validating software is quite similar, i.e. the development costs are fixed. Where a software house 'makes their money', is at the estimation stage; a good estimate ensures commercial profitability, a poor estimate realizes a commercial loss. The construct developed herein, presents a robust \& flexible TEE Model, cognizant of commercial realities. 


\section{Shaping The Solution}

\subsection{The Big Picture}

A key message this construct conveys is that specific levels of Quality Assurance (QA) incur specific costs. That is, the greater the required level of Cyber-Confidence in the solution, the greater the associated cost of QA. The mathematics underlying the presented formulation is commonplace in many fields of endeavor \& widely understood. At a fundamental level, the presented scientific solution is not new, only its application in this instance. A primary motivation for the approach formulated is to mitigate the compound estimation error typically associated with estimates constructed in the testing space, on the back of development effort estimates. Moreover, the absence of detailed information relevant to the Software-Testing-Life-Cycle (STLC) compounds the difficulty of robust test estimation. The Test Effort Estimation (TEE) Model formulated herein, assists to define a ceiling on the anticipated test effort required by a project or operations team, in-line with business objectives \& target delivery dates via the CyberRisk Management Processes articulated in the preceding sections. This approach empowers clients to precisely specify whatever level of Cyber-Risk their business is capable of absorbing, or prepared to absorb. To facilitate the TEE Model formulation process, we shall draw upon physical modeling techniques employed by other engineering disciplines such as free body diagrams \& mechanical representations. The advantages associated with our TEE Model Construct are stated in Tab. (4) as follows;

\begin{tabular}{|l|l|}
\hline \multicolumn{2}{|c|}{ Advantages } \\
\hline 1 & Requires minimal inputs \\
\hline 2 & Possesses a scientific foundation \\
\hline 3 & Facilitates operational decision-making \\
\hline 4 & Quantifies Risk Based Testing (RBT) via geometric properties \\
\hline 5 & Simple, robust, flexible, consistent, reusable \& transparent \\
\hline 6 & Capable of scaling a projected solution from a known solution \\
\hline 7 & Embraces Continuous Improvement Processes (CIP's) \\
\hline 8 & Confines perceptual subjectivity predominantly to three variables \\
\hline
\end{tabular}

Tab. (4): TEE Model Advantages

Common TEE techniques incorporate the derivation of projections based upon a specific metric such as 'Requirements', Lines-of-Code (LoC), Function Point's, combinational methods or manning ratios. The main restriction with these approaches is that they rely upon a frame of reference being well understood \& relatively stable. In the case of 'Requirements' for example, all parties involved in the Software-Development-LifeCycle (SDLC) have differing notions of what constitutes a 'Requirement'; e.g. a sales executive is unencumbered by detail \& may view 'fly to the moon' as being the detailed articulation of a requirement to 'leave The Earth'. Subsequently, in the absence of a homogeneous End-to-End (E2E) definition of 'what a requirement is', the application of 'Requirements' for the purposes of test estimation is limited. In fact, one may argue that 'Requirements' are only a valid frame of reference with respect to estimation for an immediately proceeding stage of the SDLC. Moreover, the utilization of combinational methods requires precise \& exact knowledge of the number of features to be tested (e.g. checkboxes, radio buttons, dropdown menus etc.). Unfortunately, the acquisition of the raw information required for such approaches leaps substantially beyond the confines \& 
capabilities of a potential solution provider at early stage engagements. Commonly, the prospective solution provider is not supplied the granular level of detail necessary to employ these methodologies with any measure of self-confidence.

\section{Quality Assurance \& Cyber-Risk}

Risk is a measure of uncertainty \& an inherent attribute of many virtual \& physical systems. Indeed, even physical mechanisms at the quantum level of reality are governed by Heisenberg's Uncertainty Principle; i.e. the limit of accuracy with which certain pairs of physical properties may be simultaneously known. Hence, the need to understand \& accept the existence of Cyber-Risk is fundamental to minimizing the test effort required to Quality Assure complex information systems. It is simply impractical \& inappropriate to deny the existence of Risk, as this undermines the physical nature of the Universe. Consequently, the best we can hope to achieve is to manage an agreed level of acceptable Cyber-Risk, \& mitigate unacceptable Cyber-Risk by validating software 'smarter' not 'harder'. For example, the number of possible Functional Process pair permutations forming a single Business Process from a population of 100 Functional Processes may be written \& computed according to Eq. (2) as follows;

$$
\operatorname{PERMUT}(100,2)=9,900
$$

Hence, it is clear that when just two Functional Processes (from 100) combine (on average) to form a testable Business Process, the potential cost of exhaustive validation approaches impracticality very rapidly; for coupled sets of three (3), four (4) or five (5) Functional Processes, the number of permutations expands to:

- $\operatorname{PERMUT}(100,3)=970,200$

- $\operatorname{PERMUT}(100,4)=94,109,400$

- $\operatorname{PERMUT}(100,5)=9,034,502,400$

Thus, an element of Cyber-Risk Management must be incorporated into any pragmatic solution or tool. The preceding mathematical example emphasizes a case of practical impossibility for testing all permutations. Therefore, we have incorporated a mathematical function into our construct to circumvent the practical inability of testing impossibly large permutations of potential information pathways, or any other environmental attribute beyond our immediate influence or control. The limitations associated with finite testing were incorporated into our construct by formalizing a mathematical expression for Quality as a function of Cyber-Risk.

\section{Validation}

\subsection{The Scientific Method}

The scientific method relies exclusively upon validation \& replication for its selfcorrecting properties. In particular, replication of experimental results by independent sources is vitally important \& its absence ensures that the scientific method is not selfcorrecting. Similarly, validation without replication is meaningless, as results that cannot be replicated by independent researchers, cannot be summarily validated; hence, validation \& replication are self-reinforcing features of the scientific method. 
Unfortunately in the Information Technology (IT) space, the scientific method is not enforced as rigorously as in the physical sciences, \& claims that would not be published in renowned physical science literature are frequently published in IT journals.

\subsection{User Validation}

Our Test Effort Estimation (TEE) Model Construct is in commercial use \& has been validated via the historical application of datasets across numerous projects; however, due to commercial confidence the results cannot be placed in the public domain. Even though the results could be masked to avoid identification \& preserve client confidentiality, a User could not replicate the results because they are project specific. Indeed, the many authors whom publish unverifiable results are in fact asking the readership to accept their results on-faith. This is quite a troublesome fact, proliferated by 'trust' in the name of the institution/s \&/or the station of the author/s.

However, our TEE Model Construct offers a departure from this practice, in favour of a User Validation (UV) mechanism whereby readers can validate our TEE Model Construct against their own datasets \& the experimental replication requirement associated with the scientific method is preserved. In other words, everything required by readers to valid our TEE Model Construct, is self-contained within this article \& references.

\subsection{Experimental Validation}

Our Test Effort Estimation (TEE) Model Construct has been experimentally verified to Predict \& Measure Cyber-Risk to high precision \& accuracy [>98.07\%] [1], [2].

\subsection{Demonstrational Case Study}

A Case Study demonstrating how our Test Effort Estimation (TEE) Model Construct was applied commercially to Manage Cyber-Risk, illustrating [3]:

1. How we delivered a project $30 \%$ cheaper \& $15 \%$ faster without increasing human resourcing levels

2. How Over-Testing \& Under-Testing occurs

3. How Quality-Gaps are formed

\section{Discussion}

The need for accurate Test Effort Estimation (TEE) techniques to Predict \& Measure Cyber-Risk cannot be overstated. The software testing function may consume a significant proportion of overall budget, even on well-governed Information Technology (IT) projects. With significant interests \& finite financial resources at stake, a genuine need exists for the reliable $\&$ accurate prediction of the required test effort. For solution providers in the business of 'winning business', particularly fixed-price, commercial profitability is predominantly confined to the projected costs contained in the bid submission. The situation faced by software houses is akin to a common business model adopted by speculative homebuilders in Australia. The builder generates the majority of profit from the purchase price of the land, not from the construction of the dwelling itself. Constructions costs are considered 'fixed', with trivial variation over short periods 
of time. The builder relies heavily upon the margin gained at land purchase to realise the greatest profit. Software solution providers face an analogous situation, whereby for fixed-price solutions, the cost of the build is not likely to vary significantly over the expected life of the project. Hence, the instant at which the solution provider generates their greatest profitability occurs at the estimation phase of the project. If the estimates are well constructed \& accurate, the venture is maximally profitable. If on the other hand the estimates are poorly formulated, the solution provider may face significant financial losses under a fixed-price business model. Hence, the need for accuracy, consistency \& transparency is organisationally vital.

Two well-formalised off-the-shelf TEE methodologies are Combinatorics \& CoSMIC. Combinatorics is defined as the branch of mathematics studying the enumeration, combination, \& permutation of sets of elements \& the mathematical relations that characterize their properties [14]. In terms of TEE, Combinatorics involves the quantification of the combinations of Function Point testing \& may be loosely described as a 'test everything' approach. A major proponent of Combinatorics testing is the National Institute of Standards \& Technology (NIST) [15], [16]. NIST claims that empirical evidence demonstrates that most failures are triggered by one or two parameters, \& progressively fewer by three, four, or more parameters, a relationship that they call the Interaction Rule:

1. This claim does nothing to quantify the residual Cyber-Risk associated with any level of testing. Discovering defects because they can be found, does not address the issue of Undiscovered Defects in any quantifiable manner, unless the CyberRisk Prediction \& Measurement Construct presented herein is utilized. Hence, based upon the Interaction Rule alone, one cannot quantifiably determine when the testing function can or should cease

Moreover, they also claim that if all faults in a system can be triggered by a combination of ' $n$ ' or fewer parameters, then testing all ' $n$-way' combinations of parameters can provide high confidence that nearly all faults have been discovered:

2. This is a difficult claim to accept given the absence of any technique to numerically quantify the confidence (i.e. Cyber-Confidence) to which they refer. No equivalent to Eq. (1) is presented, hence it is unclear as to how the claim of 'high confidence' may be scientifically supported

In addition, NIST state that Combinatorics can detect hard-to-find software faults more efficiently than manual test case selection methods. Although this may be a useful methodology, Combinatorics has some significant limitations. Firstly, the purpose of software testing is not to find defects, the purpose of testing software is to provide an agreed \& acceptable level of Quality Assurance (QA) to the customer; by analogy, a drug company does not measure success by the number of patients it kills or sickens using its products:

3. Finding software faults is only half the story that needs to be told. Predicting the probability of Undiscovered Defects (i.e. the Cyber-Risk) is the other half of the story. In the absence of both halves of the story, the Cyber-Risk Management decision of when to cease testing can not be executed in any meaningful scientific manner or context

If a solution provider is implementing an off-the-shelf product with customised code to be overlayed, finding defects in an out-of-the-box product may not be an economically wise undertaking. Rather, it is commercially more attractive to confine the defect- 
finding exercise to the customised portions of the code. Commercial organisations do not test MS-Word or MS-Excel if they are adopted as standard tools; they will tend to only test the customised features (if any) which may have been written for their specific needs. Microsoft invests a vast sum of money testing the core functionality of these products \& there is little sense in finding defects so deep within the code, that it benefits Microsoft \& not the needs of the customer. Moreover, if one carefully scrutinises Combinatorics, one finds that it requires a far more advanced level of application \& business knowledge than is available at the early stages of a project or commercial engagement. Thus, Combinatorics is a useful tool, but it is a limiting approach to apply in situations where a service provider is in the process of 'winning the business'.

A collective known as the Common Software Measurement International Consortium (CoSMIC) has developed a functional size measurement methodology they claim is the most advanced method available for measuring the functional size of software [17]. They state that the purpose of the CoSMIC method is to provide a standardized approach for measuring the functional size of software from Functional Domains. The CoSMIC method was accepted by ISO / IEC JTC1 SC7 in December 2002 as International Standard ISO / IEC 19761 Software Engineering - CoSMIC-FFP - a functional size measurement method. Although we do not conceptually disagree with the CoSMIC approach, the commercial reality of winning business means that CoSMIC methodology is too exhaustive to be employed at the early stages of project engagements, when insufficient information is available from client sources to execute the CoSMIC method. This should not be taken to imply that the CoSMIC approach is invalid, however, CoSMIC requires a far greater level of maturity in terms of foundational knowledge than early stages of engagements permit. In order to address the lack of available information at early stages, another approach is required in order to Predict \& Measure Cyber-Risk; i.e. The TEE Model Construct presented herein.

Our TEE Model Construct addresses \& solves a number of significant fundamental issues with respect to estimating the test effort required on software projects in order to Predict \& Measure Cyber-Risk. The greatest challenge facing TEE, particularly at the commercial bidding stage, is the lack of knowledge associated with the scope of testing itself. Many common estimation models require a vastly superior body of knowledge in order to be applied at such an early stage of the Software-Development-Life-Cycle (SDLC). Estimation techniques relying upon combinatorial considerations are fundamentally flawed for application at early stage engagements, primarily due to the absence of confirmed Function Point's, definable Functional Process Pathways \& screen layouts. Without a known number of Function Point's per screen, it is impossible to compute the potential combinations to test. Moreover, without a precise \& confirmed number of screens containing these Function Point's, combinatorial approaches to TEE are highly problematic. Indeed, in many situations, clients simply do not know \& are unable to clearly articulate the Function Point content of screens, thus rendering combinatorial approaches inert. Often, clients rely completely upon the service provider to define the scope of testing, rather than the reverse; unfortunately, this is a commercial reality with little hope of reversal without a dramatic cultural shift in the marketplace. Consequently, an urgent \& real need exists for an estimation technique, freed from the shackles of clear 'Requirements' definition at such an early stage of the SDLC. 
Estimating test effort based upon 'Requirements' is an equally flawed approach to the estimation function because they (Requirements) often mean different things to different people. In fact, even throughout the SDLC the constitution of a 'Requirement' changes with time, business need, resourcing, Scope/Statement of Work (SoW) \& comprehension. Medium to large consulting practices routinely encounter the provision of thousands of 'Requirements' from a prospective client, but these are often deemed unusable or too High-Level for the purposes of formulating a reliable response. Given the commercial consequences of 'getting it wrong' \& with only a small submission contingency being permissible common business practice, the need for a scientifically based estimation model is accentuated. Moreover, the growing commercial practice of fixed-price solutions emphasises the imperative for accurate, consistent \& responsive TEE.

Experiential estimation techniques also radiate a spectrum of problems as individual experiences may vary significantly. Estimating experientially within a collective often brings tremendous advantages, however, the estimates generated by the collective may be significantly biased by the seniority of specific individuals within the collective; thereby, potentially introducing social motivations into the submission, simply from the perspective of human nature. Whilst relying upon experience is a completely natural act, it is not necessarily summarily accurate. By analogy, centuries ago, human experience dominated physical perceptions \& the world was believed to be completely flat. Of course, we live in an enlightened age \& we now know that this experientially based conclusion is factually incorrect. Therefore, a useful way forward may be to augment experience with scientifically based argument.

The supplementary files containing experimental data associated with this article are:

1. Supplementary File: Experimental Evidence [1]

2. Supplementary File: Engineering Model [2]

3. Supplementary File: Demonstrational Case Study [3]

\section{Conclusion}

We have precisely \& unambiguously mathematically defined Cyber-Risk utilising Eq. (1). This yielded an experimentally validated solution demonstrating 'How to Predict \& Measure Cyber-Risk' for any Internet Connected Information System (ICIS) to greater than $98.07 \%$ accuracy. Moreover, we have shown that our solution holds for all scales of ICIS, from an Application level to an Enterprise level. In addition, we have shown that Test Effort Estimation (TEE) quantifies Cyber-Confidence, which in turn quantifies Cyber-Risk. Hence, TEE is a Mission Critical Activity (MCA) when formulating CyberRisk Management Strategies \& may be utilised prior to project commencement, in-flight or post facto as an assessment \&/or auditing tool.

\section{Our TEE Model Construct:}

1. Is a statistical based methodology whereby the evaluations/decisions made, result in the contraction or expansion of the ' $z$-Score' associated with an infinite population of database records. The primary advantage of this approach is that very little information is required client-side at the engagement stage in order to 
produce peer acceptable estimates of the required test effort, \& to accurately predict \& measure the associated Cyber-Risk.

2. Empowers clients \& service providers to precisely define whatever level of Cyber-Risk is to be contractually delivered, capable of being absorbed, or prepared to be absorbed by consensus. With the aid of a decision table, estimators are able to articulate \& convey to the appropriate authorities, various levels of Cyber-Risk commensurate with the available resources.

3. Presents an experimentally verified methodology, cognizant of commercial realities, yielding the following key advantages; (i) it requires minimal inputs, (ii) it has a scientific foundation, (iii) it facilitates operational decision-making, (iv) it quantifies Risk Based Testing (RBT), (v) it is simple, robust, flexible, consistent, reusable \& transparent, (vi) it is capable of scaling a projected solution from a known solution, (vii) it embraces Continuous Improvement Processes (CIP's), (viii) it confines perceptual subjectivity predominantly to three variables \& (ix), it commercially exists as an off-the-shelf product.

\section{References}

[1] Supplementary File: Experimental Evidence:

- https://www.researchgate.net/publication/344084557_How_to_Predict_Measur e_Cyber-Risk_PoC

[2] Supplementary File: Engineering Model:

- https://www.researchgate.net/publication/344085430_The_TEE_Test_Effort_Es timation_Model

[3] Supplementary File: Demonstrational Case Study:

- https://www.researchgate.net/publication/344084762_Demonstrational_Cyber -Risk_Case_Study

[4] Sharma S., Vijayvargiya S. (2021) Applying Soft Computing Techniques for Software Project Effort Estimation Modelling. In: Nath V., Mandal J. (eds) Nanoelectronics, Circuits and Communication Systems. Lecture Notes in Electrical Engineering, vol 692. Springer, Singapore:

- https://link.springer.com/chapter/10.1007/978-981-15-7486-3_21

[5] AGS: a precise and efficient AI-based hybrid software effort estimation model (Ananth \& Shrinivasan: 2020): International Journal of Business Intelligence and Data Mining (IJBIDM), Vol. 18, No. 1, 2021:

- $\quad$ https://doi.org/10.1504/IJBIDM.2021.111739

[6] Sinha R.R., Gora R.K. (2021) Software Effort Estimation Using Machine Learning Techniques. In: Goar V., Kuri M., Kumar R., Senjyu T. (eds) Advances in Information Communication Technology and Computing. Lecture Notes in Networks and Systems, vol 135. Springer, Singapore:

- https://link.springer.com/chapter/10.1007/978-981-15-5421-6_8

[7] Wińska E., Kot E., Dąbrowski W. (2021) Reducing the Uncertainty of Agile Software Development Using a Random Forest Classification Algorithm. In: Przybyłek A., Miler J., 
Poth A., Riel A. (eds) Lean and Agile Software Development. LASD 2021. Lecture Notes in Business Information Processing, vol 408. Springer, Cham:

- $\quad$ https://link.springer.com/chapter/10.1007/978-3-030-67084-9_9

[8] Pandey, M., Litoriya, R. \& Pandey, P. Validation of Existing Software Effort Estimation Techniques in Context with Mobile Software Applications. Wireless Pers Commun 110, 1659-1677 (2020):

- https://link.springer.com/article/10.1007/s11277-019-06805-0

[9] Asad Ali, Carmine Gravino, Improving software effort estimation using bio-inspired algorithms to select relevant features: An empirical study, Science of Computer Programming, Volume 205, 2021, 102621, ISSN 0167-6423:

- $\quad$ https://doi.org/10.1016/j.scico.2021.102621

[10] Altaleb, Abdullah, Rashed, Altherwi, Muna and Gravell, Andrew (2020) An industrial investigation into effort estimation predictors for mobile app development in agile processes. In ICITM 2020 - 2020 9th International Conference on Industrial Technology and Management. IEEE. pp. 291-296:

- $10.1109 /$ ICITM48982.2020.9080362

[11] López-Martín, C. Machine learning techniques for software testing effort prediction. Software Qual J (2021):

- https://link.springer.com/article/10.1007/s11219-020-09545-8

[12] Saini J.R., Chomal V.S. (2020) A Double-Weighted Parametric Model for Academic Software Project Effort Estimation. In: Fong S., Dey N., Joshi A. (eds) ICT Analysis and Applications. Lecture Notes in Networks and Systems, vol 93. Springer, Singapore:

- $\quad$ https://link.springer.com/chapter/10.1007/978-981-15-0630-7_4

[13] Sergio Di Martino, Filomena Ferrucci, Carmine Gravino, Federica Sarro, Assessing the effectiveness of approximate functional sizing approaches for effort estimation, Information and Software Technology, Volume 123, 2020, 106308, ISSN 0950-5849:

- https://doi.org/10.1016/j.infsof.2020.106308

[14] http://mathworld.wolfram.com/Combinatorics.html

[15] https://csrc.nist.gov/projects/automated-combinatorial-testing-for-software

[16] Combinatorial Software Testing:

- https://www.researchgate.net/publication/224574583_Combinatorial_Software _Testing

[17] CoSMIC: https://cosmic-sizing.org 